\title{
Application of a next-generation sequencing (NGS) panel in newborn screening efficiently identifies inborn disorders of neonates
}

\author{
Xinwen Huang ${ }^{1 \dagger}$, Dingwen $\mathrm{Wu}^{1,4+}$, Lin Zhu² ${ }^{2}$ Wenjun Wang ${ }^{2}$, Rulai Yang ${ }^{1}$, Jianbin Yang ${ }^{1}$, Qunyan $\mathrm{He}^{3}$, \\ Bingquan Zhu ${ }^{1,5}$, Ying You ${ }^{3}$, Rui Xiao ${ }^{3^{*}}$ and Zhengyan Zhao ${ }^{1,6^{*}}$ (D)
}

\begin{abstract}
Background: Newborn screening (NBS) has been implemented for neonatal inborn disorders using various technology platforms, but false-positive and false-negative results are still common. In addition, target diseases of NBS are limited by suitable biomarkers. Here we sought to assess the feasibility of further improving the screening using nextgeneration sequencing technology.

Methods: We designed a newborn genetic sequencing (NBGS) panel based on multiplex PCR and next generation sequencing to analyze 134 genes of 74 inborn disorders, that were validated in 287 samples with previously known mutations. A retrospective cohort of 4986 newborns was analyzed and compared with the biochemical results to evaluate the performance of this panel.

Results: The accuracy of the panel was $99.65 \%$ with all samples, and 154 mutations from 287 samples were 100\% detected. In 4986 newborns, a total of 113 newborns were detected with biallelic or hemizygous mutations, of which 36 newborns were positive for the same disorder by both NBGS and conventional NBS (C-NBS) and 77 individuals were NBGS positive/C-NBS negative. Importantly, 4 of the 77 newborns were diagnosed currently including 1 newborn with methylmalonic acidemia, 1 newborn with primary systemic carnitine deficiency and 2 newborns with Wilson's disease. A total of 1326 newborns were found to be carriers with an overall carrier rate of $26.6 \%$.

Conclusion: Analysis based on next generation sequencing could effectively identify neonates affected with more congenital disorders. Combined with C-NBS, this approach may improve the early and accurate identification of neonates with inborn disorders. Our study lays the foundation for prospective studies and for implementing NGS-based analysis in NBS.
\end{abstract}

Keywords: Newborn genetic screening, Inherited disorders, Multiple PCR, Gene mutation

*Correspondence: xiaorui@biosan.cn; zhaozy@zju.edu.cn

tXinwen Huang and Dingwen Wu have contributed equally to the work.

${ }^{1}$ Department of Genetics and Metabolism, Children's Hospital

of Zhejiang University School of Medicine, National Clinical Research Center for Child Health, Hangzhou, People's Republic of China

${ }^{3}$ Zhejiang Biosan Biochemical Technologies Co. Ltd, 859 Shixiang West Rd, Hangzhou 310007, Zhejiang Province, People's Republic of China

Full list of author information is available at the end of the article

\section{Introduction}

Newborn screening (NBS) is one of the most successful public health initiatives for preventing disability and death. Since the first NBS was started in the 1960s to test for phenylketonuria (PKU), diseases included in NBS have been expanded extensively [1,2]. The introduction of tandem mass spectrometry (MS/MS) is one of the most important advances in NBS, because it allows more original author(s) and the source, provide a link to the Creative Commons licence, and indicate if changes were made. The images or other third party material in this article are included in the article's Creative Commons licence, unless indicated otherwise in a credit line to the material. If material is not included in the article's Creative Commons licence and your intended use is not permitted by statutory regulation or exceeds the permitted use, you will need to obtain permission directly from the copyright holder. To view a copy of this licence, visit http://creativecommons.org/licenses/by/4.0/. The Creative Commons Public Domain Dedication waiver (http://creativeco mmons.org/publicdomain/zero/1.0/) applies to the data made available in this article, unless otherwise stated in a credit line to the data. 
than 40 metabolic disorders to be detected from dried blood spot (DBS) samples [3, 4].

Although previous screening methods featured simple, rapid, and convenient procedures, limitations also exist because metabolites or enzymatic activities are often influenced by multiple factors $[5,6]$. Some disorders, such as hearling loss, do not develop evident symptoms during the regular NBS time window; therefore, these disorders could not be detected readily by current NBS methods [7]. Analysis of disease-associated DNA molecules or genes could be a great supplemental technology for conventional screening, particularly for the disorders without specific metabolites or biomarkers such as severe combined immune deficiency (SCID) and spinal muscular atrophy (SMA) [8-10].

A variety of DNA-based techniques have been applied in NBS of specific disorders. Recent development and rapid implementation of next-generation sequencing (NGS) in the molecular diagnosis of genetic disorders provide further options and opportunities for DNAbased NBS, as exemplifed by NGS-based genetic analysis as a regular diagnostic work-up following an abnormal NBS result $[4,11]$. For disorders with clearly defined pathogenic variations, genetic screening can be used in first-tier tests of SCID [8, 12], XLA [9], SMA [10, 13], fragile $\mathrm{X}$ syndrome $[14,15]$ and hearing loss [16]. It has been reported that genetic testing as second-tier analyses improves the diagnostic specificity of CF [17], MMA [18], DMD [19] and NICCD [20]. In 2013, four genetic screening projects (BabySeq/NBSeq/NC NEXUS/STATseq) based on NGS to explore the clinical utility of whole exome sequencing (WES) and whole genome sequencing (WGS) were implemented in NBS. Although genomewide genetic screening provides information for significantly more genetic disorders, the interpretation and reporting of genomic variants remain challenging, such as the heteroplasmy of mitochondrial variants and the unknown significance (VUS) variants. In addition, WES and WGS are still relatively expensive and the amount of DNA from dried blood is limited for sequencing library preparation [21]. Consequently, WES/WGS were not considered suitable for first-tier testing, but could be of merit as second-tier testing according to the programs [11, 22-24]. A more efficient and less expensive approach is warranted for sequence analysis of DNA from DBS to screen for congenital disorders in neonates.

In the present study we designed a newborn genetic sequencing (NBGS) panel based on multiplex PCR to analyze sequences of 134 genes of 74 neonatal inborn disorders that were validated with 287 samples that have molecular diagnosis previously. Furthermore, retrospective studies were performed with 4986 archived DBS for exploring a potential screening strategy that combines conventional NBS (C-NBS) with genetic screening of neonatal inborn disorders, as well as increasing the capacity to screen out diseases not contained in C-NBS.

\section{Materials and methods Study subjects}

A total of 287 samples with molecular diagnoses from previous genetic analyses were enrolled in the validation cohort to validate the performance of the NBGS panel. The retrospective cohort includes two groups of 4986 samples in total, from neonates born between January 2017 and December 2019, who received C-NBS, including inherited metabolic disease screening by MS/ MS methods, and congenital hypothyroidism $(\mathrm{CH})$ and glucose-6-phosphate dehydrogenase deficiency (G6PDD) screening by biochemical methods. Of the 4986 samples, group 1 of 2973 were randomly selected from those with a negative initial C-NBS results, and group 2 of 2013 were from samples with a positive result in the initial C-NBS, which received repeat MS/MS analysis. All 4986 samples were analyzed with NBGS, of which 5 samples were excluded from data analysis due to low quality of NGS data and 4981 NGS result were included in the following analysis. In group 2, 53 newborns were C-NBS true positive as confirmed by repeat C-NBS and by clinical manifestation, 8 samples were lost to follow-up, 5 infants with unexplained death, and 1947 were negative by repeat C-NBS and were denoted as MS/MS false-positive cases. The potential capacity of NBGS to reduce C-NBS falsepositives and C-NBS false-negatives was estimated in 1947 MS/MS false-positive newborns and the remaining newborns that were undiagnosed clinically, respectively. Also, the value of NBGS to screen disorders out of C-NBS was evaluated in these newborns. The C-NBS result, hearing screening outcome and clinical profiling including newborn gestational age (GA, in weeks), birth weight (BW, in grams) were collected from all newborns. This study was approved by the institutional review board of the ethics committee in Children's Hospital, Zhejiang University School of Medicine.

\section{Selection of disorders, genes and variants}

Seventy four inborn disorders were included in this panel based on the Secretary's Advisory Committee on Heritable Disorders in Newborns and Children and the Wilson and Jungner criteria $[25,26]$, disorders that can be detected by biochemical test were selected with consideration of Recommended Uniform Screening Panel (RUSP) recommendation, and disease with high incidences but cannot be screened by C-NBS in China were also enrolled. The disorders in this panel consist of inherited metabolic diseases screened by MS/MS, G6PD, CH and genetic diseases with high incidences and severe 
impact on children's health (Additional file 1: Table S1), including 39 inborn metabolic disorders screened by MS/ MS and 35 disorders of skeletal system diseases, hematological system diseases, lysosomal storage disease, etc., of which 33 disorders are not covered by C-NBS. A total of 134 genes were included according to relative prevelance in associated 74 disorders. Known pathogenic variants in 134 genes (Additional file 2: Table S2) were selected as targets of the panel according to one of the following criteria: (1) high frequency in Chinese, Eastern Asian or Asian population; (2) common pathogenic variants in databases including ClinVar and ClinGen; (3) loss of function (LOF) variants in Asian population ( $\geq 10$ allele count in Gnomad) (4) high frequency in local databases. A multiplex PCR panel was designed with each selected variant covered by at least one amplicon.

\section{Library construction and sequence analysis}

Genomic DNA were extracted from dried blood spots by Nucleic Acid Automatic Extraction System (Bioer, China). DNA libraries were prepared based on multiplex PCR by using the SLIMamp (StemLoop Inhibition Mediated amplification) method [27]. High-throughput sequencing was performed with Illumina NextSeq 500 according to the manufacturer's instruction.

\section{Bioinformatic analysis}

Raw sequencing data were processed using general procedures. Low-quality sequencing reads were removed and the reads were mapped to the NCBI human reference genome (hg19/GRCh37). After variant calling and annotation, potential pathogenic variants were kept for further evaluation. In addition to the pre-selected pathogenic variants, pathogenicity of all other variants were evaluated according to ACMG sequence variant interpretation guideline [28]. Only pathogenic and likely pathogenic variants were considered for reporting.

\section{IEM panel}

Inborn errors of metabolism (IEM) panel is an in-house targeted NGS panel of 86 genes (PAH, PTS, MUT, $S L C 22 A 5$, etc.) associated with inherited metabolic disorders. Coding exons of target genes were captured using an Agilent High Sensitivity DNA Kit (Agilent, Santa Clara, CA, USA). Libraries generated from enriched DNA were sequenced using the Illumina NextSeq 500 platform (Illumina Inc., San Diego, CA, USA) in pairedend mode.

\section{Statistical analysis}

The comparison of the biochemical index, GA and BW of different groups was done by analysis of variance
(ANOVA), and statistical analysis between any two groups was performed using a t-test (two-tailed).

\section{Result}

\section{Evaluation of the performance of NBGS panel}

In the validation cohort of 287 samples, 155 mutations of 35 genes were covered in the NBGS. All mutations in 35 genes were identified by NBGS except for one variant in the $M M A C H C$ gene (c.482G > A) in one sample due to low data quality. Overall, the accuracy of the NBGS was $99.65 \%$ (286/287). A total of 33 mutations were detected in the $P A H$ gene, followed by 18 mutations in the $S L C 22 A 5$ gene and 10 mutations in the $M M U T$ gene (Additional file 1: Fig. S1).

\section{Newborn genetic screening of the retrospective cohort}

A cohort of 4986 newborns were subjected to NBGS analysis (5 samples with low-quality data were excluded) and compared with C-NBS result (Fig. 1a). The work flow of NBGS was shown in Fig. 1b. The characteristics of all newborns were shown in Table 1 , and a small number of preterms born at 32-37 weeks were included in our study, which make up the majority of newborns with low birth weight (1500-2500 g).

In the $53 \mathrm{C}$-NBS true positive subjects, who received positive repeat $\mathrm{C}$-NBS results, 36 cases were identified as positive by NBGS, and the detection rate was $68 \%$ (36/53) (Fig. 1a, Fig. 2a), the biochemical results and genotypes were shown in Table 2. Importantly, all newborns with positive C-NBS results indicating G6PDD and tetrahydrobiopterin deficiency (BH4D) were confirmed by NBGS. For seventeen NBGS negative samples, only one mutation related to the diagnosed disease were detected in 11 newborns whereas no mutations were detected in 6 subjests (Fig. 2a). Subsequently, the NBGS negative samples were subjected to IEM panel for further analysis and biallelic or hemizygous mutations in the disorder-related genes were identified in 13 subjects (Additional file 1: Table S3).

Moreover, 13 newborns including 7 lost to followup infants and 6 unknown death cases were suspected positive by C-NBS but were identified as negative by NBGS. Further analysis was performed by IEM panel, and 2 of the lost to follow-up subgroup were identified biallelic mutations in CPS1 and ACADSB gene in two subjects with clinical diagnosis of carbamoyl phosphate synthetase I deficiency and 2-methylbutyryl-CoA dehydrogenase deficiency respectively (Additional file 1: Table S4).

A total of 77 newborns from C-NBS false-positive (57/1947, 2.9\%) and C-NBS negative (20/2973, 0.7\%) group were identified as positive by NBGS (Fig. 2b): 48 of 77 newborns suggested to have metabolic disorders by 


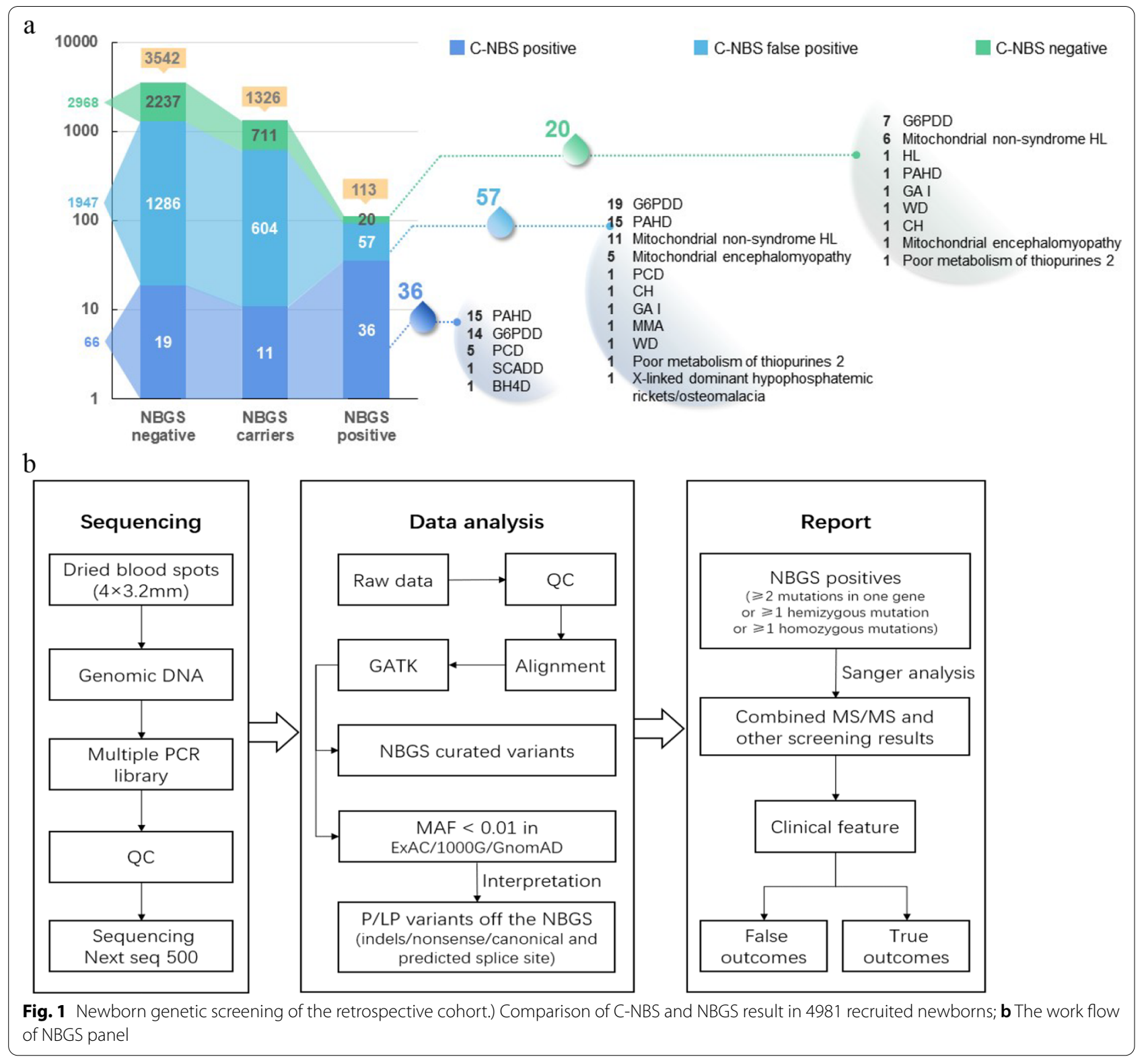

NBGS (Table 3), including 26 female with normal biochemical results with heterozygous G6PD mutations, 16 newborns with elevated primary MS/MS screening results suggestive of mild hyperhenylalaninemia with biallelic mutations in the $P A H$ gene, 2 newborns with biallelic mutations in the $G C D H$ gene for $\mathrm{GA} \mathrm{I}, 2 \mathrm{CH}$ also identified positive by NBGS and the TSH concentration was normal after followed up, and significantly 1 PCD and 1 MMA were identified positive by NBGS, which was consistent with the followed-up biochemical results, indicating a false-negative C-NBS finding at primary screening (Table 4). The remaining 29 newborns were found to carry biallelic or hemizygous mutations causing hearing loss and 4 disorders were not included in C-NBS (mitochondrial myopathy, Wilson's disease (WD), purine disorder, and rickets) (Additional file 1: Table S5), 2 of them with biallelic [c.2333G $>\mathrm{A} / \mathrm{c} .3532 \mathrm{~A}>\mathrm{G}$ ] and homozygous [c.3859G >A/c.3859G >A] mutations of $A T P 7 B$ gene were diagnosed with WD after followed up (Table 4).

\section{Detection of recessive disease carriers by NBGS}

In the 4981 newborns, heterozygous carriers were frequently observed. Except for one individual carrying heterozygous mutations in 4 genes, most carrier newborns were found to have one mutation (Fig. 3a), whereas 184 $(\sim 3.7 \%)$ newborns were found to be carriers of two or 
Table 1 The demographic characteristic of all newborns in this study

$\mathrm{N}(\%) /$ Median/Mean

\begin{tabular}{ll} 
& $\mathbf{N}(\%) /$ Median/Mean \\
\hline Gender & \\
Male & $2635(52.8 \%)$ \\
Female & $2351(47.2 \%)$ \\
Birth weight & \\
Mean & $3264 \pm 539 \mathrm{~g}$ \\
Median & $3330 \mathrm{~g}$ \\
$<1000 \mathrm{~g}$ & $6(0.1 \%)$ \\
1000-1500 g & $64(1.3 \%)$ \\
1500-2500 g & $301(6.1 \%)$ \\
2500-4000 g & $4300(86.2 \%)$ \\
$>4000 \mathrm{~g}$ & $315(6.3 \%)$ \\
Gestational age & \\
Mean & $38 \pm 2.5$ weeks \\
Median & 39 weeks \\
$<28$ weeks & $9(0.2 \%)$ \\
28-32 weeks & $101(2 \%)$ \\
32-37 weeks & $376(7.5 \%)$ \\
37-42 weeks & $4494(90.2 \%)$ \\
$>42$ weeks & $6(0.1 \%)$ \\
C-NBS true positive & 53 \\
Lost to follow-up & 7 \\
Unknown death & 6 \\
C-NBS false positive & 1947 \\
C-NBS negative & 2973 \\
Total & 4986 \\
\hline & \\
\hline & \\
\hline &
\end{tabular}

more variants in different genes. In summary, a total of 1326 newborn carriers were identified through NBGS analysis with an overall carrier frequency of $26.6 \%$ (1326/4981). The top five genes with the highest carrier frequency in these newborns were DUOX2 (14.06\%), SLC22A5 (10.54\%), GJB2 (10.47\%), ATP7B (8.5\%) and $P A H$ (7.8\%) (Fig. 3b). Genes with at least one carrier identified were listed in Additional file 1: Table S6, and five genes (MTR, TG, GBA, MTHFR and CPT2) of NBGS were associated with susceptibility to certain conditions that have summarized in Additional file 1: Table S7.

In addition, carrier newborns with ASS1 and MCCC1 mutations were noticed to have elevated levels of corresponding metabolites from C-NBS results. We compared 34 ASS1 carriers and 17 MCCC1 carriers with negative controls and a significant difference was found between carrier and control groups (Fig. 3c, d).

\section{Correlation of gestational age, birth weight, MS/MS and NBGS results}

A total of 1947 newborns were false-positive by MS/MS primary screening, of which 1890 cases were identified as negative by NBGS consistent with follow-up testing. To further explore the possible factors related to inconsistent results of primary MS/MS screening and NBGS, GA and BW were analyzed in diagnosed newborns (NBGS positive), C-NBS false-positive newborns (MS/MS primary positive and NBGS negative) and control group (healthy newborns with negative C-NBS and NBGS results). The results showed that newborns in the C-NBS false positive group are associated with significantly lower BW or smaller GA, suggesting low BW and small GA might affect levels of metabolites resulting in falsepositive outcomes (Additional file 1: Fig. S2a, S2b). Low BW and small GA newborns with false-positive conventional screening were further divided to subgroups based on BW (<1000 g; 1000-1500 g; 1500-2500 g) and GA newborns (<28 weeks, 28-32 weeks, 32-37 weeks), and no significant difference was found between each groups.

\section{Discussion}

The compelling problems facing NBS include reducing false-positive and false-negative results, improving the positive detection rate and expanding the spectrum of screened disorders beyond current C-NBS methods [20, 29-34]. We adapted a validated genetic screening panel for newborn genetic screening to achieve the goal. Our results showed that NBGS could reliably detect targeted variants, identify newborns affected by conditions not included in current C-NBS and reduce false-positive rate by C-NBS in our recruited population that highlight the complementarity of both methods.

In C-NBS true-positive newborns, 68\% (36/53) of them were identified as positive by NBGS. Several studies have demonstrated the diagnostic rates of NGS panels for IEM-affected infants were approximately 50-59\% $[35,36]$. However, a recent study showed that WES had an overall sensitivity of $88 \%$ for infants who screened positive by C-NBS, in which $32 \%$ of the reported variants were absent from both HGMD and ClinVar [37], consistent with the sensitivity of the NBGS panel that only reports known mutations. NBGS showed good performance in disorders with high incidence such as G6PDD (100\%) and phenylalanine hydroxylase deficiency (PAHD) (83.3\%), similar to the detection rates reported in other studies [32, 38, 39]. For the 17 C-NBS true-positive/NBGS negative cases, the possible reasons include: (1) $\mathrm{CH}$ in our study were all identified negative by NBGS, it was likely that the $\mathrm{CH}$ associated gene mutation identified in few patient, and genetic mechanism responsible remains to be elucidated, only $51.82 \%(57 / 110) \mathrm{CH}$ patients have been reported carried biallelic mutations of 21 candidate genes [40]. Due to limited genes and hotspot mutations, false-negative result would occur in genetic screening and it was suggested that the conventional 


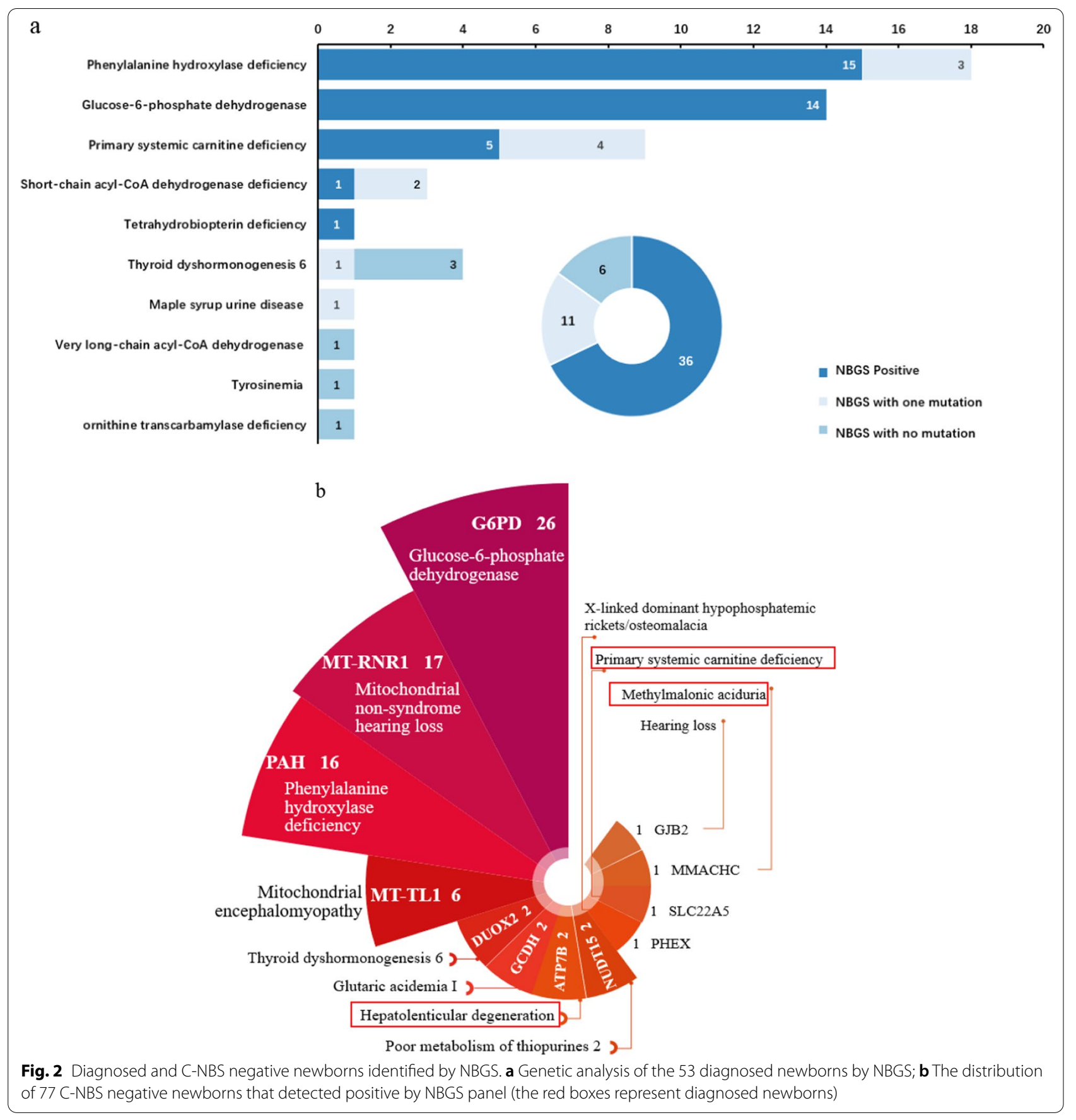

screening is more suitable for $\mathrm{CH}$ in this study. (2) The NBGS negative P/LP variants or disorder were not targeted in our panel. (3) The VUS variants detected by IEM panel were not included in NBGS because the unclear mechanism and interpretation of VUS was challenging for clinicians in a screening panel. As literature and clinical data evolve, the VUS may become reportable and the panel should be reassessed by then. (4) Larger copy-number variants (CNVs) could not be identified by NBGS. (5)
The mechanism of genetic disease was not defined completely and the relevant genes may be undiscovered, and 11 cases were also negative after performed IEM in this study.

In the 77 C-NBS false-positive and false-negative/ NBGS positive infants, G6PDD was the most prevalent condition, followed by hearing loss and PAHD. Female carriers of heterozygous G6PD mutations are at elevated risk of developing acute hemolysis, but could be 
Table 2 The C-NBS true positive newborns identified positive by NBGS

\begin{tabular}{|c|c|c|c|c|c|}
\hline NO & Conditions (Gene) & Biochemical range & Positive rule & NBGS result & Cases \\
\hline 1 & PAHD $(P A H)$ & $\begin{array}{l}\text { PHE: } 120-769 \mu \mathrm{mol} / \mathrm{L} \\
\text { PHE/TYR: } 1.2-12\end{array}$ & $\begin{array}{l}\mathrm{PHE}>100 \mu \mathrm{mol} / \mathrm{L}, \\
\mathrm{PHE} / \mathrm{TYR}>1.2\end{array}$ & $\begin{array}{l}{[c .1068 \mathrm{C}>\mathrm{A} / \mathrm{c} .505 \mathrm{C}>\mathrm{A}] ;[\mathrm{c} .688 \mathrm{G}>\mathrm{A} / \mathrm{c} .688 \mathrm{G}>\mathrm{A}] ;} \\
{[\mathrm{c} .728 \mathrm{G}>\mathrm{A} / \mathrm{c} .728 \mathrm{G}>\mathrm{A}] ;[\mathrm{c} .722 \mathrm{delG} / \mathrm{c} .466 \mathrm{G}>\mathrm{C}] ;[\mathrm{c} .1216 \mathrm{~A}>\mathrm{G} / \mathrm{c} .8 \mathrm{C}>\mathrm{T}] ;} \\
{[\mathrm{c} .721 \mathrm{C}>\mathrm{T} / \mathrm{c} .721 \mathrm{C}>\mathrm{T}] ;[\mathrm{c} .194 \mathrm{~T}>\mathrm{C} / \mathrm{c} .158 \mathrm{G}>\mathrm{A}] ;[\mathrm{c} .975 \mathrm{C}>\mathrm{G} / \mathrm{c} .158 \mathrm{G}>\mathrm{A}] ;} \\
{[\mathrm{c} .1068 \mathrm{C}>\mathrm{A} / \mathrm{c} .158 \mathrm{G}>\mathrm{A}] ;[\mathrm{c} .1162 \mathrm{G}>\mathrm{A} / \mathrm{c} .158 \mathrm{G}>\mathrm{A}] ;} \\
{[\mathrm{c} .331 \mathrm{C}>\mathrm{T} / \mathrm{c} .158 \mathrm{G}>\mathrm{A}] ; 2^{*}[\mathrm{c} .728 \mathrm{G}>\mathrm{A} / \mathrm{c} .158 \mathrm{G}>\mathrm{A}] ;} \\
{[\mathrm{c} .1252 \mathrm{~A}>\mathrm{C} / \mathrm{c} .158 \mathrm{G}>\mathrm{A}] ;[\mathrm{c} .838 \mathrm{G}>\mathrm{A} / \mathrm{c} .1123 \mathrm{C}>\mathrm{G}] ;}\end{array}$ & 15 \\
\hline 2 & G6PDD (G6PD) & G6PD/6PGD: $0.3-0.9$ & G6PD/6PGD < 1.0 & $\begin{array}{l}\text { Male } \\
5^{*}[\mathrm{c} .1466 \mathrm{G}>\mathrm{T}] ; 3^{*}[\mathrm{c} .1478 \mathrm{G}>\mathrm{A}] \\
2^{*}[\mathrm{c} .1114 \mathrm{C}>\mathrm{T}] ; 2^{*}[\mathrm{c} .185 \mathrm{~A}>\mathrm{G}] \\
\text { Female } \\
{[\mathrm{c} .185 \mathrm{~A}>\mathrm{G}] ;[\mathrm{c} .1388 \mathrm{G}>\mathrm{A}]}\end{array}$ & 14 \\
\hline 3 & PCD (SLC22A5) & $\mathrm{C} 0: 7-7.3 \mu \mathrm{mol} / \mathrm{L}$ & $\mathrm{CO}<9.5 \mu \mathrm{mol} / \mathrm{L}$ & $\begin{array}{l}2^{*}[\mathrm{c} .760 \mathrm{C}>\mathrm{T} / \mathrm{C} .1400 \mathrm{C}>\mathrm{G}] ;[338 \mathrm{G}>\mathrm{A} / \mathrm{c} .1400 \mathrm{C}>\mathrm{G}] ; \\
{[\mathrm{c} .428 \mathrm{C}>\mathrm{T} / \mathrm{c} .1400 \mathrm{C}>\mathrm{G}] ;[\mathrm{c} .497+1 \mathrm{G}>\mathrm{T} / \mathrm{c} .760 \mathrm{C}>\mathrm{T}]}\end{array}$ & 5 \\
\hline 4 & SCADD (ACADS) & $\mathrm{C} 4=1.14 \mu \mathrm{mol} / \mathrm{L}$ & $\mathrm{C} 4>0.7 \mu \mathrm{mol} / \mathrm{L}$ & c. $164 C>T / c .1130 C>T$ & 1 \\
\hline 5 & $\begin{array}{l}\text { Tetrahydrobiop- } \\
\text { terin deficiency } \\
\text { (PTS) }\end{array}$ & $\begin{array}{l}\mathrm{PHE}=318 \mu \mathrm{mol} / \mathrm{L} \\
\mathrm{PHE} / \mathrm{TYR}=4.2\end{array}$ & $\begin{array}{l}\mathrm{PHE}>100 \mu \mathrm{mol} / \mathrm{L}, \\
\mathrm{PHE} / \mathrm{TYR}>1.2\end{array}$ & c. $317 C>T / c .84-291 A>G$ & 1 \\
\hline Total & & & & 36 & \\
\hline
\end{tabular}

Table 3 The biochemical range and NBGS result of 48 C-NBS false positive and C-NBS negative newborns with variants related to metabolic disorders

\begin{tabular}{|c|c|c|c|c|c|}
\hline NO & Conditions (Gene) & Biochemical range & Positive value & NBGS result & Cases \\
\hline \multirow[t]{3}{*}{1} & G6PDD & G6PD: 30.6-44 U/dL & $\mathrm{G} 6 \mathrm{PD}<26 \mathrm{U} / \mathrm{dL}$ & Female & 26 \\
\hline & $(G 6 P D)$ & G6PD: $2.3-5.8 \mathrm{u} / \mathrm{gHb}$ & or G6PD $<2.6 \mathrm{u} / \mathrm{gHb}$ & $5^{*}[\mathrm{c} .185 \mathrm{~A}>\mathrm{G}] ;[\mathrm{c} .406 \mathrm{C}>\mathrm{T}] ; 4^{*}[\mathrm{c} .1376 \mathrm{G}>\mathrm{T}] ;$ & \\
\hline & & & & $\begin{array}{l}8^{*}[\mathrm{c} .1388 \mathrm{G}>\mathrm{A}] ;[\mathrm{c} .871 \mathrm{G}>\mathrm{A}] ;[\mathrm{c} .1360 \mathrm{C}>\mathrm{T}] ; 2^{*} \\
{[\mathrm{c} .392 \mathrm{G}>\mathrm{T}] ;[\mathrm{C} .1004 \mathrm{C}>\mathrm{A}] ; 3^{*}[\mathrm{C} .1024 \mathrm{C}>\mathrm{T}] ;}\end{array}$ & \\
\hline \multirow[t]{6}{*}{2} & PAHD $(P A H)$ & PHE: $102-162 \mu \mathrm{mol} / \mathrm{L}$ & $\mathrm{PHE}>100 \mu \mathrm{mol} / \mathrm{L}$ & {$[c .1256 \mathrm{~A}>\mathrm{G} / \mathrm{c} .158 \mathrm{G}>\mathrm{A}] ;[\mathrm{c} .721 \mathrm{C}>\mathrm{T} / \mathrm{c} .158 \mathrm{G}>\mathrm{A}]$} & 16 \\
\hline & & & & $\begin{array}{l}{[\mathrm{c} .728 \mathrm{G}>\mathrm{A} / \mathrm{c} .158 \mathrm{G}>\mathrm{A}] ;[\mathrm{c} .331 \mathrm{C}>\mathrm{T} / \mathrm{c} .158 \mathrm{G}>\mathrm{A}]} \\
{[\mathrm{c} .320 \mathrm{~A}>\mathrm{G} / \mathrm{c} .158 \mathrm{G}>\mathrm{A}] ; 2^{*}} \\
{[\mathrm{c} .1197 \mathrm{~A}>\mathrm{T} / \mathrm{c} .158 \mathrm{G}>\mathrm{A}]}\end{array}$ & \\
\hline & & & & {$[c .611 \mathrm{~A}>\mathrm{G} / \mathrm{c} .158 \mathrm{G}>\mathrm{A}] ;[$ [c.1199G $>$ A/c.158G $>\mathrm{A}]$} & \\
\hline & & & & {$[c .964 G>A / c .158 G>A] ;[c .1068 C>A / c .158 G>A]$} & \\
\hline & & & & $\begin{array}{l}{[c .4421 \mathrm{G}>\mathrm{A} / \mathrm{c} .158 \mathrm{G}>\mathrm{A}] ; 2^{*}} \\
{[\mathrm{c} .842+2 \mathrm{~T}>\mathrm{A} / \mathrm{c} .158 \mathrm{G}>\mathrm{A}]}\end{array}$ & \\
\hline & & & & $[\mathrm{c} .721 \mathrm{C}>\mathrm{T} / \mathrm{c} .158 \mathrm{G}>\mathrm{A}] ;$; c.208_210del/c.158G $>\mathrm{A}] ;$ & \\
\hline 3 & GA I (GCDH) & $\mathrm{C} 5 \mathrm{DC}+\mathrm{C} 6-\mathrm{OH}: 0.07-0.18 \mu \mathrm{mol} / \mathrm{L}$ & $\mathrm{C} 5 \mathrm{DC}+\mathrm{C} 6-\mathrm{OH}>0.4 \mu \mathrm{mol} / \mathrm{L}$ & $2^{*}[\mathrm{c} .1244-2 \mathrm{~A}>\mathrm{C} / \mathrm{c} .1261 \mathrm{G}>\mathrm{A}]$ & 2 \\
\hline 4 & PCD (SLC22A5) & $\mathrm{CO}=10.5 \mu \mathrm{mol} / \mathrm{L}$ & $\mathrm{C} 0<9.5 \mu \mathrm{mol} / \mathrm{L}$ & C. $760 C>T / C .1400 C>G$ & 1 \\
\hline \multirow[t]{2}{*}{5} & MMA (MMACHC) & $\mathrm{C} 3=3.5 \mu \mathrm{mol} / \mathrm{L}$ & $\mathrm{C} 3>4 \mu \mathrm{mol} / \mathrm{L}$ & c.609G $>\mathrm{A} / \mathrm{c} .617 \mathrm{G}>\mathrm{A}$ & 1 \\
\hline & & $C 3 / C 2=0.18$ & $\mathrm{C} 3 / \mathrm{C} 2>0.25$ & & \\
\hline 6 & $\mathrm{CH}(\mathrm{D} \cup \mathrm{O} \times 2)$ & TSH: $2.5-5.9 \mu \mathrm{IU} / \mathrm{mL}$ & $\mathrm{TSH}>9.0 \mu \mathrm{IU} / \mathrm{mL}$ & $\begin{array}{l}{[c .1588 \mathrm{~A}>\mathrm{T} / \mathrm{c} .1588 \mathrm{~A}>\mathrm{T}] ; \text { [ }\left[\mathrm{c} .2104 \_2106 \mathrm{del} /\right.} \\
\text { c.2104_2106del] }\end{array}$ & 2 \\
\hline Total & & & & 48 & \\
\hline
\end{tabular}

difficult to recognize by conventional biochemical methods. It has been reported that female carriers identified by genetic screening showed mild phenotypes, which is also observed in our study [41]. Therefore, combined biochemical methods with genetic screening could improve the identification of G6PDD. Mitochondrial 12S rRNA mutations are related to aminoglycoside induced deafness [42]. The mutation frequencies of mtDNA m.1557A > G and $\mathrm{m} .1496 \mathrm{C}>\mathrm{T}$ in China were $0.20 \%$ and $0.03 \%$ respectively [43]. Genetic screening helps to identify carriers and avoid deafness caused by administration of aminoglycoside antibiotics. For all of 16 PAHD newborns identified by NBGS, the c.158G > A variant was found in trans with a pathogenic or likely pathogenic variant in the PAH gene. The c.158G > A variant has been reported as a pathogenic variant in hyperphenylalaninemia patients, 
Table 4 Summary of clinical and genetic features of 4 diagnosed newborns by NBGS

\begin{tabular}{|c|c|c|c|c|}
\hline & Case NP0467 & Case NP0865 & Case NP0956 & Case NP0439 \\
\hline Gender & Male & Female & Female & Male \\
\hline Age of onset & 14 months & 3 years old & 14 months & 3 years old \\
\hline \multirow[t]{6}{*}{ Biochemical result } & Primary screening result: & & N/A & N/A \\
\hline & $\mathrm{C} 3=3.5 \mu \mathrm{mol} / \mathrm{L}$ & $\mathrm{C} 0=10.5 \mu \mathrm{mol} / \mathrm{L}$ & & \\
\hline & $C 3 / C 2=0.18$ & & & \\
\hline & Follow-up result: & & & \\
\hline & $\mathrm{C} 3=5.2 \mu \mathrm{mol} / \mathrm{L}$ & $\mathrm{CO}=7.3 \mu \mathrm{mol} / \mathrm{L}$ & & \\
\hline & $C 3 / C 2=0.34$ & & & \\
\hline Birthweight (g) & 3650 & 2700 & 2900 & 3660 \\
\hline Gestational age (weeks) & $39+2$ & $39+0$ & $38+3$ & $39+2$ \\
\hline Diagnosed disease & Methylmalonic aciduria & $\begin{array}{l}\text { primary systemic carnitine } \\
\text { deficiency }\end{array}$ & Wilson's disease & Wilson's disease \\
\hline Gene & MMACHC & SLC22A5 & $A T P 7 B$ & $A T P 7 B$ \\
\hline Genotype & $\mathrm{c} .609 \mathrm{G}>\mathrm{A} / \mathrm{c} .617 \mathrm{G}>\mathrm{A}$ & c. $760 \mathrm{C}>\mathrm{T} / \mathrm{C} .1400 \mathrm{C}>\mathrm{G}$ & c. $2333 G>A / c .3532 A>G$ & c.3859G $>$ A/c.3859G $>A$ \\
\hline
\end{tabular}

N/A, not applicable

however, this variant was also considered as "likely benign" due to high population frequency and clinical evidence [44]. The concentration of phe elevated slightly at regular intervals and no clinical intervention was performed in 16 PAHD. It seems that c.158G > A may relate to mild hyperphenylalaninemia in our enrolled population. The most common mutation of mitochondrial encephalomyopathy is m.3224A $>\mathrm{G}$, however, classical presentations may be absent in some pediatric patients [45]. Because of the heteroplasmy, it is difficult to evaluate the mutation load in related tissues/organs, thereby making diagnosis more challenging. A total of six newborns were identified with carriers of m.3224A $>\mathrm{G}$ by NBGS, we have been followed up these cases until now, and all without any clinical manifestation, maybe it suggested that mitochondrial disorders were not suitable for newborn genetic screening.

Using NBGS, 4 cases were diagnosed with MMA, PCD and WD that had been missed by C-NBS. It has been reported that the determination of MMA by MS/MS has been affected by several factors, and one case was missed due to the concentrations of $\mathrm{C} 3$ and $\mathrm{C} 3 / \mathrm{C} 0$ decrease with age $[46,47]$. However, unlike metabolites, genetic screening was sufficiently accurate and specific for inborn disorders because it does not vary with age, season or maternal status. WD was not screened in C-NBS due to unavailability of specific markers. Some asymptomatic patients do not receive effective treatment before irreversible injury is present [48]. In our study, 2 newborns showed normal liver function initially but identified positive by NBGS. Both newborns were diagnosed, and the old sister of NP0439 also was determined soon. It was note-worthy that applied genetic screening to C-NBS could effectively reduce the false-negative cases of C-NBS, and also could identify an additional subgroup of patients that undetectable by C-NBS, and the turn-around time was also decreased compared with conventional method that at the time of their clinical presentation arisen, actually, the turn-around time of C-NBS methods would takes about 1-2 months, one with positive initial screening result and positive multiple retest needs other biochemical test and genetic analysis to confirm. However, the testing period of NBGS is just 10 working days and C-NBS result was also finished during the time, combined NBGS and traditional methods, the turn-around time would decreased to 2-3 weeks. Moreover, expanding the spectrum of disorders that can not be screened by MS/MS could be highly beneficial especially for those with high incidence, treatable and early diagnosis is helpful for their prognosis. In our panel, a total of 33 disorders out of C-NBS could be identified such as WD, SCID and XLA.

Recently, the carrier rate of multiple disorders had been mainly reported in carrier screening of the general population. The carriers for at least one disorder was reported to be $24 \%$ [49], as in our study also showed about $1 / 3$ screened individuals with at least one pathogenic mutation in childhood diseases. It was challenging to provide genetic counseling for such a large amount of all infants but that is meaningful to carriers of genetic disorders to determine risk for future offspring and their parents to have additional children. In the future prospective study, we would offer an "opt-out" option for reporting carrier state when signed informed consent of NBGS testing and the related counseling. If the parents choose to report carrier result and the infant identified as carrier, we can provide genetic counseling for them. The top genes such 


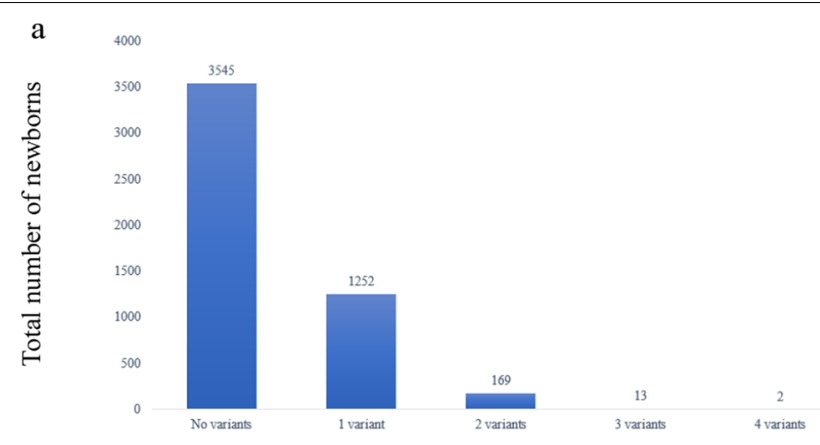

b 250
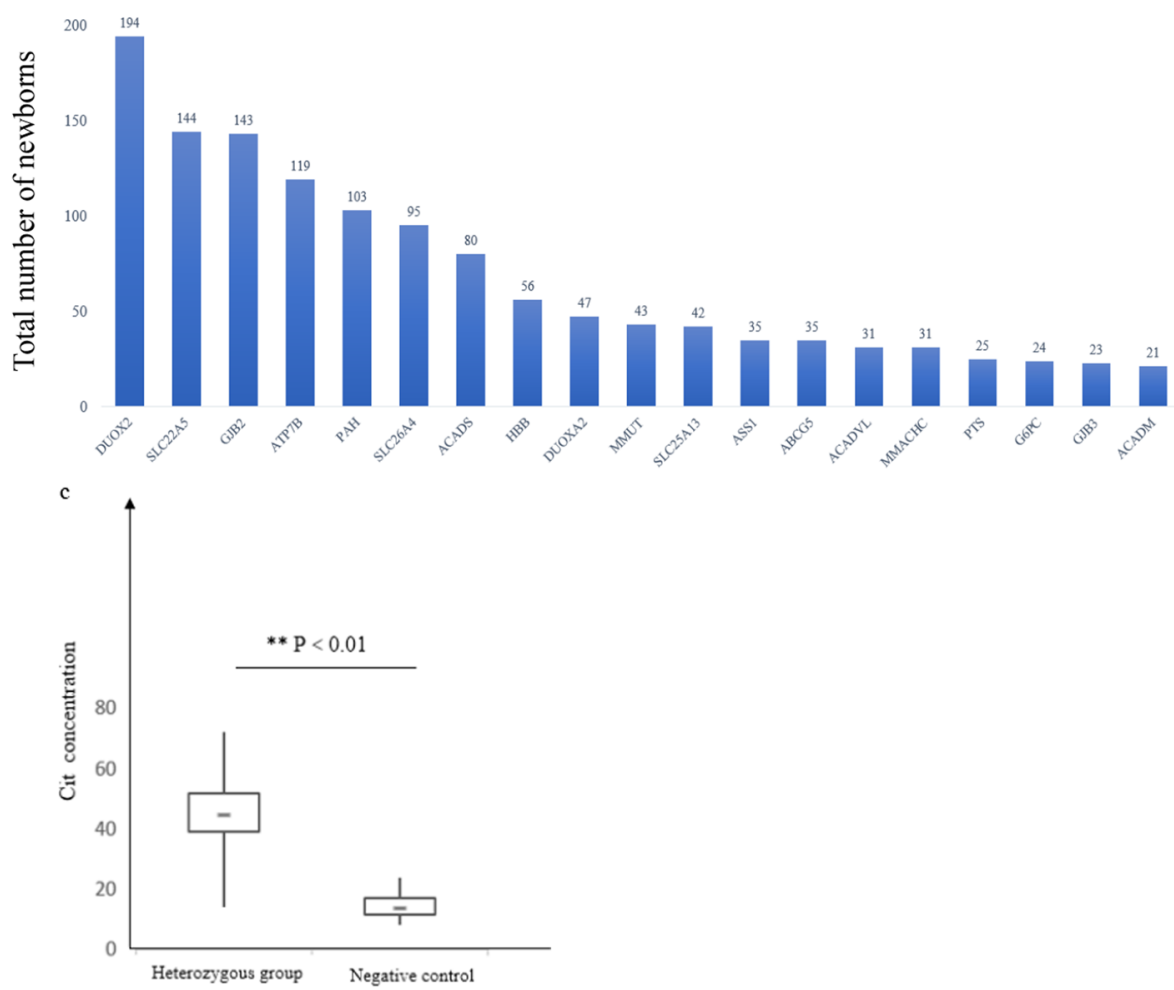

ASSI Carriers

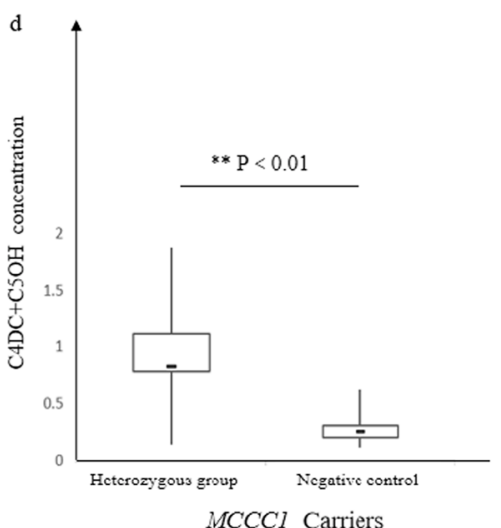

Fig. 3 The mutation frequency in carriers and biochemical index of ASS1 and MCCC1 carriers. a The numbers of variants in carriers. $\mathbf{b}$ The distribution of high frequency gene mutations in carriers. c Cit concentration in ASS1 gene mutation carriers and negative control. $\mathbf{d}$ C4DC $+\mathrm{C} 5 \mathrm{OH}$ concentration in $\mathrm{MCCC} 1$ gene mutation carriers and negative control 
as PAH, SLC22A5, ACADS and MUT were most frequently reported for carriers known to have high carrier frequency in reported study [46], which could verify the relative high incidence of associated inborn disorders [50, 51]. Some high-frequency mutations of $D U O X 2, H B B$, $A T P 7 B$ gene in our study were not found in previous investigations. This may be attribute to the differences in targeted population, sample size, genetic background, and detected range of mutations $[47,50]$. In addition, although NBGS panel was designed to screen neonates, reporting increased risk for certain conditions may be beneficial, particularly for carrier parents. However, we would recommend additional consents and counseling for reporting these conditions since uncertainty of disease onset and reduced penetrance could cause unnecessary burden for participants and change the risk-benefit balance.

In 1890 C-NBS false-positive/NBGS negative newborns, carriers with ASS1 and MCCC1 mutation showed abnormal biochemical results, but all newborns with normal phenotype until now. Generally, it is unlikely that variants of carrier would impact phenotype. A previous study showed $4.3 \%$ heterozygous carriers with mild presentations such as in Hemophilia A, CH, and G6PDD [41]. Given the limited numbers in carriers with phenotype and the limited understanding of their penetrance and functional studies, the relationship of carrier status and phenotypic expression is still ambiguous [41]. Therefore, NBGS could help to exclude some false positives and to reduce follow-up time and anxiety of heterozygous carriers. Moreover, we also observed GA and BW with significant difference in false-positive newborns and control. The physiological states associated with preterm, LBW, and sick newborns and the treatments received directly affect the reliability of results for many diseases screened in public health [52-54]. Metabolite levels were influenced by birth weight and gestational age $[6,55]$, which might increase false positives of C-NBS but could screened out by NBGS. In low BW and small GA newborns, we have not found difference in each subgroups which maybe due to the small amount of preterm infants in our study.

In summary, our results showed that advantages of the NBGS panel as a novel newborn screening test may include: (1) analysis for more preventable and treatable disorders that are not targeted by C-NBS; (2) preselected and pre-curated mutations that avoid complex and cumbersome interpretation and reporting; (3) lowercost and faster turn-around time compared with WES or capture-based NGS panels that need more complex experimental process and data analysis; (4) providing rapid diagnosis of affected newborns after screened positive by C-NBS and improving the cost-effectiveness; (5) reducing false-positive newborns identified by C-NBS. Our data suggest that it may be a more effective strategy for newborn screening to combine NBGS with C-NBS. However, the NBGS test does have limitations. Because the NBGS panel only covers pre-selected known mutations, it will inevitably miss some pathogenic variants, which may lead to false-negative results and low overall detection rates. Although capture-based NGS methods could provide almost complete coverage of coding regions of targeted genes, variants in noncoding regions such as introns and untranslated regions are usually not readily detected, as well as large copy number variations (CNV) and complex structural variations (SV). In addition, because the NBGS test only reports pathogenic and likely pathogenic variants, a significant number of rare variants, particularly missense and splicing variants, will be intepreted as VUS due to lack of reported clinical or research evidence, and thus not reported, as indicated in a recent study [37]. Nonetheless, if C-NBS and NBGS are concurrently applied, joint ananlysis of biochemical and sequence data would help optimize both tests reciprocally. Further research is needed to evaluate the clinical utility and effectiveness in a larger newborn population to explore the long-term implication of sequencingbased newborn screening.

\section{Conclusions}

In this study, we have developed an NGS panel test based on multiplex PCR with low cost, fast turn-around-time and great specificity for newborn genetic screening of 74 disorders. Our data suggest that NBGS combined with C-NBS could improve screening efficiency, reduce the false-positive/-negative results, and also expand the spectrum of screened disorders to achieve the purpose of early detection and diagnosis.

\section{Abbreviations}

NBS: Newborn screening; MS/MS: Tandem mass spectrometry; DBS: Dried blood spots; NBGS: Newborn genetic sequencing; C-NBS: Conventional newborn screening.

\section{Supplementary Information}

The online version contains supplementary material available at https://doi. org/10.1186/s13023-022-02231-x.

Additional file 1: Table S1, S3, S4, S5, S6, S7, Figure S1, S2.

Additional file 2: Table 52

\section{Acknowledgements}

We thank sincerely for all the clinical and experiments staff from neonatal screening center and department of genetics and metabolism of Children's Hospital of Zhejiang University School of Medicine, bioinformatics analysis by yuan-yuan Liang and technical staff from Zhejiang Biosan Biochemical Technologies Co. Ltd and Hangzhou Biosan Clinical Laboratory Co. Ltd. 


\section{Authors' contributions}

$X W H, Y Y, Z Y Z$ conceived and designed the work; DWW, QYH performed the experiments, XWH, DWW, RLY, JBY and QBZ analyzed the clinical data, $\mathrm{LZ}$ and WJW analyzed the sequencing data, XWH, LZ, WJW and RX wrote the manuscript. All authors read and approved the final manuscript.

\section{Funding}

This work was supported by the National Key Research and Development Program of China (Grant Number 2018YFC1002200)

\section{Availability of data and materials}

The datasets analyzed during the present study are available from the corresponding author on reasonable request.

\section{Declarations}

\section{Ethics approval and consent to participate}

This study was approved by the institutional review board of the ethics committee in Children's Hospital, Zhejiang University School of Medicine.

\section{Consent for publication}

Not applicable.

\section{Competing interest}

The authors declare that they have no competing interests.

\section{Author details}

'Department of Genetics and Metabolism, Children's Hospital of Zhejiang University School of Medicine, National Clinical Research Center for Child Health, Hangzhou, People's Republic of China. ${ }^{2}$ Hangzhou Biosan Clinical Laboratory Co. Ltd, 859 Shixiang West Road, Hangzhou, Zhejiang Province, People's Republic of China. ${ }^{3}$ Zhejiang Biosan Biochemical Technologies Co. Ltd, 859 Shixiang West Rd, Hangzhou 310007, Zhejiang Province, People's Republic of China. ${ }^{4}$ Zhejiang Neonatal Screening Center, Department of Genetics and Metabolism, Children's Hospital of Zhejiang University School of Medicine, National Clinical Research Center for Child Health, Hangzhou, China. ${ }^{5}$ Department of Child Healthcare, Children's Hospital of Zhejiang University School of Medicine, National Clinical Research Center for Child Health, Hangzhou, People's Republic of China. ${ }^{6}$ Department of PediatricsChildren's Hospital of Zhejiang University School of Medicine, National Clinical Research Center for Child Health, 3333 Binsheng Rd, Hangzhou 310052, Zhejiang Province, People's Republic of China.

Received: 21 June 2021 Accepted: 6 February 2022

Published online: 21 February 2022

\section{References}

1. Rajabi F. Updates in newborn screening. Pediatr Ann. 2018;47(5):e187-90.

2. Patel H, Feldman M. Universal newborn hearing screening. Paediatr Child Health. 2011;16(5):301-10.

3. Watson MS, Mann MY, Lloyd-Puryear MA, Rinaldo P, Howell RR, American College of Medical Genetics Newborn Screening Expert Group. Newborn screening: toward a uniform screening panel and system-executive summary. Pediatrics. 2006;117(5 Pt 2):S296-307.

4. Urv TK, Parisi MA. Newborn screening: beyond the spot. Adv Exp Med Biol. 2017;1031:323-46.

5. HonzíkT, et al. Carnitine concentrations in term and preterm newborns at birth and during the first days of life. Prague Med Rep. 2005;106(3):297-306.

6. Ryckman KK, et al. Association of amino acids with common complications of prematurity. Pediatr Res. 2013;73(6):700-5.

7. Norris WW, et al. Does universal newborn hearing screening identify all children with GJB2 (Connexin 26) deafness? Penetrance of GJB2 deafness. Ear Hear. 2006;27(6):732-41.

8. de Felipe B, et al. Prospective neonatal screening for severe T- and B-lymphocyte deficiencies in Seville. Pediatr Allergy Immunol. 2016;27(1):70-7.
9. Chen XF, et al. Clinical characteristics and genetic profiles of 174 patients with X-linked agammaglobulinemia: report from Shanghai, China (2000-2015). Medicine (Baltimore). 2016;95(32):e4544.

10. Lin $Y$, et al. Newborn screening for spinal muscular atrophy in China using DNA mass spectrometry. Front Genet. 2019;10:1255.

11. Berg JS, et al. Newborn sequencing in genomic medicine and public health. Pediatrics. 2017;139(2):e20162252.

12. Borte $S$, von Döbeln U, Hammarström L. Guidelines for newborn screening of primary immunodeficiency diseases. Curr Opin Hematol. 2013;20(1):48-54

13. Kariyawasam DST, et al. The implementation of newborn screening for spinal muscular atrophy: the Australian experience. Genet Med. 2020;22(3):557-65.

14. Tassone F, et al. FMR1 CGG allele size and prevalence ascertained through newborn screening in the United States. Genome Med. 2012;4(12):100.

15. Maenner MJ, et al. FMR1 CGG expansions: prevalence and sex ratios. Am J Med Genet B Neuropsychiatr Genet. 2013;162b(5):466-73.

16. Wang $Q$, et al. Nationwide population genetic screening improves outcomes of newborn screening for hearing loss in China. Genet Med. 2019:21(10):2231-8.

17. Kloosterboer M, et al. Clarification of laboratory and clinical variables that influence cystic fibrosis newborn screening with initial analysis of immunoreactive trypsinogen. Pediatrics. 2009;123(2):e338-46.

18. Peng $\mathrm{G}$, et al. Combining newborn metabolic and DNA analysis for second-tier testing of methylmalonic acidemia. Genet Med. 2019;21(4):896-903.

19. Ke Q, et al. Newborn screening for Duchenne muscular dystrophy in China: follow-up diagnosis and subsequent treatment. World J Pediatr. 2017;13(3):197-201.

20. Lin Y, et al. Combining newborn metabolic and genetic screening for neonatal intrahepatic cholestasis caused by citrin deficiency. J Inherit Metab Dis. 2020;43(3):467-77.

21. Poulsen JB, et al. High-quality exome sequencing of wholegenome amplified neonatal dried blood spot DNA. PLoS ONE. 2016;11(4):e0153253.

22. Holm IA, et al. The BabySeq project: implementing genomic sequencing in newborns. BMC Pediatr. 2018;18(1):225.

23. Lewis MA, et al. Supporting parental decisions about genomic sequencing for newborn screening: the NC NEXUS decision aid. Pediatrics. 2016;137 Suppl 1(Suppl 1):S16-23.

24. Farnaes $L$, et al. Rapid whole-genome sequencing decreases infant morbidity and cost of hospitalization. NPJ Genom Med. 2018:3:10.

25. Wilson JM, Jungner YG. Principles and practice of mass screening for disease. Bol Oficina Sanit Panam. 1968;65(4):281-393.

26. Calonge $\mathrm{N}$, et al. Committee report: method for evaluating conditions nominated for population-based screening of newborns and children. Genet Med. 2010;12(3):153-9.

27. Schenk D, et al. Amplification of overlapping DNA amplicons in a singletube multiplex PCR for targeted next-generation sequencing of BRCA1 and BRCA2. PLOS ONE. 2017;12(7):e0181062.

28. Richards S, et al. Standards and guidelines for the interpretation of sequence variants: a joint consensus recommendation of the American College of Medical Genetics and Genomics and the Association for Molecular Pathology. Genet Med. 2015;17:405-24.

29. Rossi C, et al. A false-positive case of methylmalonic aciduria by tandem mass spectrometry newborn screening dependent on maternal malnutrition in pregnancy. Int J Environ Res Public Health. 2020;17(10):3601.

30. Weiss KJ, et al. Diagnostic challenges using a 2-tier strategy for methylmalonic acidurias: data from 1.2 million dried blood spots. Ann Nutr Metab. 2020;76(4):268-76.

31. Dorreh $\mathrm{F}$, et al. Determining the TSH reference range in national newborn screening program for congenital hypothyroidism. J Matern Fetal Neonatal Med. 2020;33(19):3244-8.

32. Liu Z, et al. Chinese newborn screening for the incidence of G6PD deficiency and variant of G6PD gene from 2013 to 2017. Hum Mutat. 2020;41(1):212-21.

33. Peng $G$, et al. Ethnic variability in newborn metabolic screening markers associated with false-positive outcomes. J Inherit Metab Dis. 2020;43(5):934-43.

34. Wu CC, et al. Newborn genetic screening for hearing impairment: a population-based longitudinal study. Genet Med. 2017;19(1):6-12. 
35. Ghosh A, et al. Diagnosing childhood-onset inborn errors of metabolism by next-generation sequencing. Arch Dis Child. 2017;102(11):1019-29.

36. Navarrete R, et al. Value of genetic analysis for confirming inborn errors of metabolism detected through the Spanish neonatal screening program. Eur J Hum Genet. 2019;27(4):556-62.

37. Adhikari AN, et al. The role of exome sequencing in newborn screening for inborn errors of metabolism. Nat Med. 2020;26(9):1392-7.

38. Chen T, et al. Mutational and phenotypic spectrum of phenylalanine hydroxylase deficiency in Zhejiang Province, China. Sci Rep. 2018:8(1):17137.

39. Yang $C$, et al. Newborn screening and diagnosis of inborn errors of metabolism: a 5-year study in an eastern Chinese population. Clin Chim Acta. 2020;502:133-8.

40. Sun F, Zhang JX, et al. The genetic characteristics of congenital hypothyroidism in China by comprehensive screening of 21 candidate genes. Eur J Endocrinol. 2018;178(6):623-33.

41. Ceyhan-Birsoy $\mathrm{O}$, et al. Interpretation of genomic sequencing results in healthy and ill newborns: results from the BabySeq project. Am J Hum Genet. 2019;104(1):76-93.

42. Usami $\mathrm{S}$, et al. Prevalence of mitochondrial gene mutations among hearing impaired patients. J Med Genet. 2000;37(1):38-40.

43. Fu Y, et al. Carrier frequencies of hearing loss variants in newborns of China: a meta-analysis. J Evid Based Med. 2019;12(1):40-50.

44. Choi $R$, et al. Reassessing the significance of the PAH c.158G >A (p.Arg53His) variant in patients with hyperphenylalaninemia. J Pediatr Endocrinol Metab. 2017;30(11):1211-8.

45. Hu C, et al. Clinical and molecular characterization of pediatric mitochondrial disorders in south of China. Eur J Med Genet. 2020;63(8):103898.

46. Hu Z, et al. Determination of methylmalonic acid, 2-methylcitric acid, and total homocysteine in dried blood spots by liquid chromatography-tandem mass spectrometry: a reliable follow-up method for propionylcarnitine-related disorders in newborn screening. J Med Screen. 2020;28(2):93-9.

47. Luo $X$, et al. A pilot study of expanded newborn screening for 573 genes related to severe inherited disorders in China: results from 1,127 newborns. Ann Transl Med. 2020;8(17):1058.

48. Ye $\mathrm{S}$, et al. Wilson disease: identification of two novel mutations and clinical correlation in Eastern Chinese patients. World J Gastroenterol. 2007;13(38):5147-50.

49. Lazarin GA, et al. An empirical estimate of carrier frequencies for $400+$ causal Mendelian variants: results from an ethnically diverse clinical sample of 23,453 individuals. Genet Med. 2013;15(3):178-86.

50. Wang T, et al. Expanded newborn screening for inborn errors of metabolism by tandem mass spectrometry in Suzhou, China: disease spectrum, prevalence, genetic characteristics in a Chinese population. Front Genet. 2019;10:1052.

51. Yang $Y$, et al. Application of next-generation sequencing following tandem mass spectrometry to expand newborn screening for inborn errors of metabolism: a multicenter study. Front Genet. 2019;10:86.

52. Hunter MK, et al. Follow-up of newborns with low thyroxine and nonelevated thyroid-stimulating hormone-screening concentrations: results of the 20-year experience in the Northwest Regional Newborn Screening Program. J Pediatr. 1998;132(1):70-4.

53. Suormala T, Wick H, Baumgartner ER. Low biotinidase activity in plasma of some preterm infants: possible source of false-positive screening results. Eur J Pediatr. 1988;147(5):478-80.

54. Marble M, et al. Neonatal vitamin B12 deficiency secondary to maternal subclinical pernicious anemia: identification by expanded newborn screening. J Pediatr. 2008;152(5):731-3.

55. Yang $L$, et al. Effects of birth weight on profiles of dried blood aminoacids and acylcarnitines. Ann Clin Biochem. 2018;55(1):92-9.

\section{Publisher's Note}

Springer Nature remains neutral with regard to jurisdictional claims in published maps and institutional affiliations.

Ready to submit your research? Choose BMC and benefit from:

- fast, convenient online submission

- thorough peer review by experienced researchers in your field

- rapid publication on acceptance

- support for research data, including large and complex data types

- gold Open Access which fosters wider collaboration and increased citations

- maximum visibility for your research: over $100 \mathrm{M}$ website views per year

At BMC, research is always in progress.

Learn more biomedcentral.com/submissions 